\title{
Dual Extended Hesitant Fuzzy Sets
}

\author{
José Carlos R. Alcantud ${ }^{1,2, * \mathbb{D}}$, Gustavo Santos-García ${ }^{2} \mathbb{D}$, Xindong Peng ${ }^{3}$ \\ (D) and Jianming Zhan ${ }^{4}$ (D) \\ 1 BORDA Research Unit, Universidad de Salamanca, 37007 Salamanca, Spain \\ 2 Facultad de Economía y Empresa and Multidisciplinary Institute of Enterprise (IME), \\ Universidad de Salamanca, 37007 Salamanca, Spain; santos@usal.es \\ 3 Department of Internet of Things, School of Information Science and Engineering, Shaoguan University, \\ Shaoguan 512005, China; pengxindong@sgu.edu.cn \\ 4 College of Science, Hubei Minzu University, Enshi 445000, China; zhanjianming@hotmail.com \\ * Correspondence: jcr@usal.es
}

Received: 4 May 2019; Accepted: 23 May 2019; Published: 26 May 2019

\begin{abstract}
Hesitant fuzzy sets extend fuzzy sets by considering many-valued sets of membership degrees. Real applications validate this model and decision making approaches of various forms permit to act in a flexible manner. If we can avail ourselves of hesitant information on non-membership degrees too, then dual hesitant fuzzy sets provide a natural extension of both hesitant fuzzy sets and intuitionistic fuzzy sets. This article defines the concept of dual extended hesitant fuzzy set as the combination of extended hesitant fuzzy sets with dual hesitant fuzzy sets. Its basic algebraic properties are set forth, and the model is linked to other successful models in the literature. We also define a comparison law for the prioritization of elements described in this new framework. Moreover, we present an algorithm to solve the dual extended hesitant fuzzy decision making problem by a weight score function. Finally, the feasibility of this approach is demonstrated by the evaluation of big data industries with an effectiveness test.
\end{abstract}

Keywords: hesitant fuzzy set; extended hesitant fuzzy set; dual hesitant fuzzy set; decision making

\section{Introduction}

Despite the success of fuzzy sets [1], imprecise information cannot always be well represented by fuzzy values. Therefore, extensions or alternative models have proliferated [2-5] (see [6] for an account of types of fuzzy sets with their relationships). In particular, there are situations in the fuzzy setting where the practitioner inputs more information than a unique membership degree, either because she receives a set of possible input values (hesitancy [7], also with necessary and possible information [8]), additional non-membership values (intuitionistic fuzzy sets [9]), or both (dual hesitant fuzzy sets [10]).

Let us dwell on the models that motivate our investigation. Hesitant fuzzy sets (HFSs) use many-valued sets of membership degrees [11-14]. Real applications validate this model, and decision-making approaches of various forms permit to act flexibly with data under hesitancy [15]. If we can also avail ourselves of hesitant information on non-membership degrees, then dual hesitant fuzzy sets (DHFSs) provide a natural extension of both HFSs and intuitionistic fuzzy sets.

In this paper, we go beyond the latter model. We are concerned with a multi-person setting. In this context we propose a rich model that permits using non-membership degrees, hesitancy (in both membership and non-membership degrees), as well as the possibility of discriminating the information depending on the person that submitted it. We achieve our purpose by a suitable hybridization of two recent extensions of HFSs, namely, extended HFSs [16] and dual HFSs. The fundamental algebraic properties of the concept that we call "dual extended hesitant fuzzy sets" are proven. We also define scores for the purpose of comparing the basic ingredients in this definition, which are extended hesitant 
fuzzy elements. We show how our model generalizes various existing models, which ensures its good performance in practice. Moreover, for solving the application of dual extended hesitant fuzzy problem, we develop a multi-attribute decision making (MADM) method based on weight score function, which is defined for that specific purpose. Finally, the effectiveness of the proposed algorithm is verified by the recourse to the criteria in Wang and Triantaphyllou [17].

This paper is organized as follows. Section 2 gives the necessary background about hesitant fuzzy sets and their extensions. Section 3 defines and develops our model, inclusive of basic algebraic properties and a comparison law, and frames it in relation with existing models. Section 4 presents a MADM method with its application in big data evaluation. We conclude in Section 5.

\section{Background about Hesitant Fuzzy Sets and Extensions}

Let us fix a non-empty set of alternatives $\mathbf{X}$. To define hesitant fuzzy set on $\mathbf{X}$, the concept of hesitant fuzzy element from Xia and $\mathrm{Xu}[18]$ is helpful:

Definition 1. A hesitant fuzzy element (HFE) is a non-empty subset of $[0,1]$. Hence, $\mathcal{P}^{*}([0,1])$ is the set of all HFEs. A typical HFE (THFE) is a non-empty, finite subset of $[0,1] . \mathcal{F}^{*}([0,1])$ denotes the set of all THFEs.

THFEs can be expressed as $h=\left\{h^{1}, \ldots, h^{l_{h}}\right\}$ with $h^{1}<\ldots<h^{l_{h}}$. In this case, $h^{-}=h^{1}$ and $h^{+}=h^{l_{h}}$ are the minimum and maximum of the HFE, respectively. In general, we define $h^{-}=\inf \{\gamma: \gamma \in h\}$ and $h^{+}=\sup \{\gamma: \gamma \in h\}$ for (non-typical) HFEs.

Several tools help the researchers to investigate the main features of HFEs. Scores are provided in [19] and the references therein. A critical review of the literature on entropy measures for HFEs is given in [20].

Definition 2. A hesitant fuzzy set on $\mathbf{X}$ is $h_{M}: \mathbf{X} \longrightarrow \mathcal{P}^{*}([0,1])$. A typical hesitant fuzzy set on $\mathbf{X}$ is $h_{M}: \mathbf{X} \longrightarrow \mathcal{F}^{*}([0,1])$.

Definition 2 is attributed to Torra [7] and Bedregal et al. [21]. Each HFS on $\mathbf{X}$ associates a set of membership values to every element of $\mathbf{X}$, and if it is typical then such set is always finite.

The HFS in Definition 2 can be expressed in a more practical notation: $M=\left\{\left(x, h_{M}(x)\right) \mid x \in \mathbf{X}\right\}$.

In typical HFSs on $\mathbf{X}$, we also write $h_{M}(x)=\left\{h_{M}^{1}(x), \ldots, h_{M}^{l_{M}(x)}(x)\right\}$ where $h_{M}^{1}(x)<\ldots<$ $h_{M}^{l_{M}(x)}(x)$. The cardinality of the HFE $h_{M}(x)$ is $l_{M}(x)$. Repetitions are excluded in $h_{M}(x)$ by definition (since it is a set).

Several recent papers give updated details about developments in the theory of HFSs [13,22-24]. In particular, we highlight that they can be hybridized with other structures. For example, hesitant fuzzy soft sets [25] arise when we import the spirit of parameterized descriptions as defined by soft sets. The concept of interval-transformed HFE [20] links HFEs and interval-valued fuzzy sets. D-intuitionistic hesitant fuzzy sets [26] combine D numbers and generalized HFSs. Hesitant probabilistic fuzzy sets $[27,28]$ incorporate probabilities corresponding to decision maker's preferences associated with feasible values in HFSs.

In the rest of this section, we proceed to give a concise introduction to some enhancements of the concept of HFS in various lines, which we need for presenting our model.

\subsection{Some Improvements of the HFS Model}

Although Torra's design of HFS has been successfully applied in many instances, it can be improved to account for a better informational basis or uncertainty in other senses beyond hesitancy:

(1) Whenever possible, it is interesting to keep track of who expressed which opinion, for example because the seniority of the agents is significant in the decision making.

(2) It is frequently the case that the agents are hesitant about membership degrees but also about the non-membership degrees of some options. 
We recall the solutions that the literature has offered to incorporate both possibilities separately in Sections 2.2 and 2.3. Then, in Section 3, we propose a novel model that simultaneously benefits from both improvements.

\subsection{Extended Hesitant Fuzzy Sets (EHFSs)}

Zhu and $\mathrm{Xu}$ [16] defined extended hesitant fuzzy sets (EHFSs) so that the values provided by the agents can be collected by value-groups. They also gave some fundamental operations for EHFEs, which are defined as the Cartesian product of HFSs. Therefore, HFSs can be used to generate EHFSs. In the other direction, particular EHFSs become HFSs. As in the case of HFSs, the constituents of the extended hesitant fuzzy sets are called extended hesitant fuzzy elements (EHFEs). The related notion of expanded hesitant fuzzy set [29] (XHFS) can be used for similar purposes.

The necessary concepts can be defined in the following way which seems more amenable than the original definition:

Definition 3. An extended hesitant fuzzy element of degree $m$ is the Cartesian product of $m$ non-empty subsets of $[0,1]$. An extended hesitant fuzzy set of degree $m$ on $\mathbf{X}$ is

$$
H_{m}^{\mathbf{X}}=\{\langle x, H(x)\rangle: x \in \mathbf{X}\} \text {, such that each } H(x) \text { is an EHFE of degree } m .
$$

The next example illustrates the ideas of EHFE and EHFS.

Example 1. Two agents give their evaluations of options $x$ and $y$.

(a) Agent 1 provides the membership set $\{0.3\}$ for $x$, and Agent 2 submits $\{0.3,0.4\}$. It corresponds to the EHFE of degree $2 H(x)=\{(0.3,0.3),(0.3,0.4)\}=\{0.3\} \times\{0.3,0.4\}$.

(b) Agent 2 provides the membership set $\{0.5,0.6\}$ for $y$, and Agent 2 submits $\{0.8\}$. It corresponds to the EHFE of degree $2 H(y)=\{(0.5,0.8),(0.6,0.8)\}=\{0.5,0.6\} \times\{0.8\}$.

Such information produces the EHFS of degree 2 on $\mathbf{X}=\{x, y\}$

$$
H_{2}^{\mathbf{X}}=\{\langle x,\{(0.3,0.3),(0.3,0.4)\}\rangle,\langle y,\{(0.5,0.8),(0.6,0.8)\}\rangle\}
$$

\subsection{Dual Hesitant Fuzzy Sets (DHFSs)}

$\mathrm{Zhu}, \mathrm{Xu}$ and $\mathrm{Xia}$ [10] introduced the following two concepts:

Definition 4. A dual hesitant fuzzy element (DHFE) is a pair $d=(h, g)$ with $h, g \subseteq[0,1]$, such that for all $\gamma \in h, \eta \in g, \gamma+\eta \leqslant 1$.

The restriction about the elements from $h$ and $g$ means that a DHFE is $d=(h, g)$ such that $h, g \subseteq[0,1]$ and $h^{+}+g^{+} \leqslant 1$ (where $h^{+}=\sup h$, and $g^{+}=\sup g$.)

Definition 5. A dual hesitant fuzzy set (DHFS) on the set $\mathbf{X}$ is $D=\{\langle x, h(x), g(x)\rangle: x \in \mathbf{X}\}$ such that each $(h(x), g(x))$ is a DHFE.

Typical DHFEs (also T-DHFEs [30]) are such that $h, g$ in Definition 4 are typical. Observe that when both $h$ and $g$ are singletons, we obtain intuitionistic fuzzy numbers [31]: see Section 3.1 of [30]. Precisely for this reason, we avoid the constraint [30] that both $h$ and $g$ should have cardinality 2 or greater. Typical DHFSs are naturally defined too [29].

In a DHFS, each agent evaluates each element by means of two respective sets of membership and non-membership values. Typically, these sets are always finite and of cardinality 2 or greater. To compare typical DHFEs, the following procedure is used [10]: 
Definition 6. Let $d_{1}=\left(h_{1}, g_{1}\right)$ and $d_{2}=\left(h_{2}, g_{2}\right)$ be typical DHFEs. Define for $i=1,2$

$$
s_{d_{i}}=\frac{\sum_{\gamma \in h_{i}} \gamma}{\left|h_{i}\right|}-\frac{\sum_{\eta \in g_{i}} \eta}{\left|g_{i}\right|}, \quad p_{d_{i}}=\frac{\sum_{\gamma \in h_{i}} \gamma}{\left|h_{i}\right|}+\frac{\sum_{\eta \in g_{i}} \eta}{\left|g_{i}\right|} \text {. }
$$

(1) When $s_{d_{1}}>s_{d_{2}}$, we say that $d_{1}$ is superior to $d_{2}$. We denote $d_{1} \succ d_{2}$.

(2) When $s_{d_{1}}=s_{d_{2}}$, we say that:

(2.1) $d_{1}$ is superior to $d_{2}$ when $p_{d_{1}}>p_{d_{2}}$. We denote $d_{1} \succ d_{2}$.

(2.2) $d_{1}$ is equivalent to $d_{2}$ when $p_{d_{1}}=p_{d_{2}}$. We denote $d_{1} \sim d_{2}$.

The researcher now has a nice toolbox to operate with this extension of HFSs. Correlation coefficients of DHFSs appear in [32-34], and we also find an application to multiple attribute decision making in [33]. Applications in pattern recognition that benefit from distance and similarity measures for DHFSs appear in [35]. Quite recently, Chen et al. [36] defined novel distances between DHFSs through the mean and standard deviations of its constituents DHFEs. Finally, Wang et al. [37] produced dual hesitant fuzzy aggregation operators and showed their applicability in MADM.

Again, the spirit of parameterized descriptions can also be merged with this model. Dual hesitant fuzzy soft sets are the outcome of this combination [38]. Their aggregation operators and corresponding applications to decision-making appear in [39].

\section{The Model: Dual Extended Hesitant Fuzzy Sets}

Motivated by the arguments in Section 2.1, we now proceed to define the natural hybridization of the notions in Sections 2.2 and 2.3 as follows:

Definition 7. A dual extended hesitant fuzzy element (DEHFE) of degree $m$ is an element $D=\left(d_{1}, \ldots, d_{m}\right)$ such that $d_{i}$ is a DHFE for $i=1, \ldots, m$. If such DHFEs are typical, then we say that the DEHFE is typical.

$A$ dual extended hesitant fuzzy set (DEHFS) of degree $m$ on the set $\mathbf{X}$ is

$$
D_{m}^{\mathbf{X}}=\left\{\left\langle x, d_{1}(x), \ldots, d_{m}(x)\right\rangle: x \in \mathbf{X}\right\},
$$

such that for each $x \in \mathbf{X}, D(x)=\left(d_{1}(x), \ldots, d_{m}(x)\right)$ is a DEHFE of degree $m$. If all such DEHFEs are typical, then we say that the DEHFS is typical.

Example 2. Two agents evaluate options $x$ and $y$ by respective DHFEs:

(a) Agent 1 provides the evaluations $d_{1}(x)=(\{0.1,0.3,0.4\},\{0.3,0.5\})$ and

$$
d_{1}(y)=(\{0.2,0.3\},\{0.4,0.5,0.6\}) .
$$

(b) Agent 2 provides the evaluations $d_{2}(x)=(\{0.2,0.5\},\{0.1,0.2,0.4\})$ and

$$
d_{2}(y)=(\{0.3,0.4,0.5\},\{0.1,0.2\}) .
$$

Such information can be collected by one item, which is the DEHFS of degree 2 on $\mathbf{X}=\{x, y\}$ defined as

$$
\begin{aligned}
D_{2}^{X}=\{\langle x,(\{0.1,0.3,0.4\},\{0.3,0.5\}),(\{0.2,0.5\},\{0.1,0.2,0.4\})\rangle \\
\langle y,(\{0.2,0.3\},\{0.4,0.5,0.6\}),(\{0.3,0.4,0.5\},\{0.1,0.2\})\rangle\}
\end{aligned}
$$

\subsection{Relationships with Existing Models}

Clearly, DEHFEs of degree 1 are DHFEs. Therefore, our model subsumes other models that were already extended by DHFEs: 
(1) When the DHFE $d=(h, g)$ is such that $|h|=|g|=1$, it is an intuitionistic fuzzy number. Therefore, DHFSs $\{\langle x, h(x), g(x)\rangle: x \in \mathbf{X}\}$ with $|h(x)|=|g(x)|=1$ for all $x \in \mathbf{X}$ are intuitionistic fuzzy sets (IFSs) [9].

(2) When the DHFE $d=(h, g)$ is such that either $h=\varnothing$ or $g=\varnothing$, it can be identified with a HFE. Therefore, DHFSs $\{\langle x, h(x), g(x)\rangle: x \in \mathbf{X}\}$ such that either $h(x)=\varnothing$ for all $x \in \mathbf{X}$, or $g(x)=\varnothing$ for all $x \in \mathbf{X}$, can be identified with HFSs.

In addition, if $D=\left(d_{1}=\left(h_{1}, g_{1}\right), \ldots, d_{m}=\left(h_{m}, g_{m}\right)\right)$ is a DEHFE of degree $m$ such that either $h_{i}=\varnothing$ for all $i$, or $g_{i}=\varnothing$ for all $i$, then $D$ can be identified with an EHFE of degree $m$. Hence, one can naturally embed EHFSs into DEHFSs too. As stated above, EHFSs of degree 1 are HFSs.

The diagram in Figure 1 summarizes these relationships.

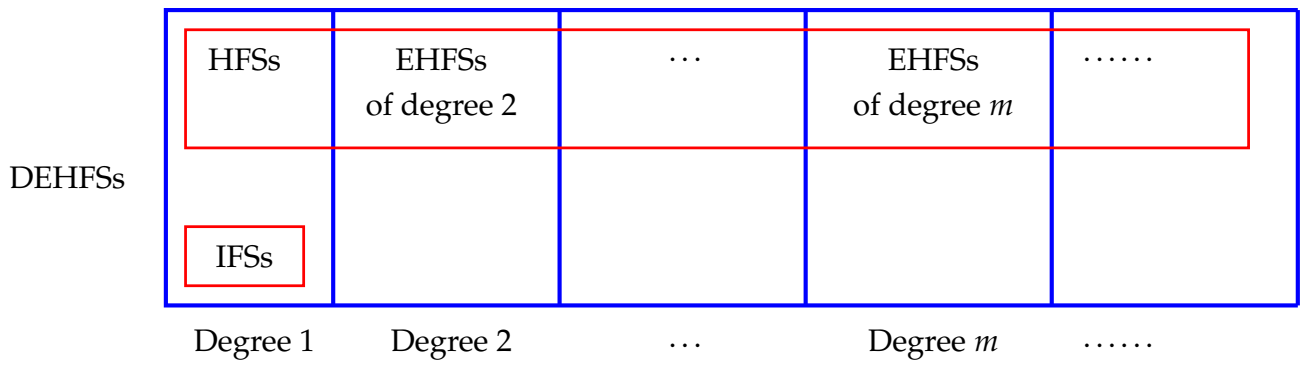

Figure 1. Relationships of DEHFSs with existing models. DEHFSs generalize IFSs, HFSs, and EHFSs of arbitrarily large degrees. See the Abbreviations list at the end of this paper.

\subsection{Algebra of Dual Extended Hesitant Fuzzy Sets}

In Section 3.2 of [10], some basic operators and operations on DHFEs are defined. We can take advantage of these elements to give a first insight into the algebra of our model.

The complement of DHFEs is an involutive operator defined as follows [10]:

$$
\text { when } d=(h, g) \text { is DHFE then } d^{c}= \begin{cases}(g, h), & \text { if } h \neq \varnothing \neq g, \\ (\{1-\gamma: \gamma \in h\}, \varnothing), & \text { if } h \neq \varnothing=g \\ (\varnothing,\{1-\eta: \eta \in g\}), & \text { if } h=\varnothing \neq g .\end{cases}
$$

We can use this operator for the following purpose:

Definition 8. Let $D=\left(d_{1}, \ldots, d_{m}\right)$ be a DEHFE of degree $m$, such that $d_{i}=\left(h_{i}, g_{i}\right)$ are DHFEs. Then, $D^{c}=\left(d_{1}^{c}, \ldots, d_{m}^{c}\right)$ is the complement of $D$.

Similarly, the complement of the DEHFS of degree $m$ on the set $\mathbf{X} D_{m}^{\mathbf{X}}=\left\{\left\langle x, d_{1}(x), \ldots, d_{m}(x)\right\rangle: x \in X\right\}$ is the DEHFE of degree $m$ given as $\left(D_{m}^{\mathbf{X}}\right)^{c}=\left\{\left\langle x, d_{1}(x)^{c}, \ldots, d_{m}(x)^{c}\right\rangle: x \in \mathbf{X}\right\}$.

The union and intersection of two DHFEs $d_{1}=\left(h_{1}, g_{1}\right)$ and $d_{2}=\left(h_{2}, g_{2}\right)$ are, respectively, defined as follows [10]:

$$
\begin{aligned}
& d_{1} \cup d_{2}=\left(\left\{\gamma \in h_{1} \cup h_{2}: \gamma \geqslant \max \left\{h_{1}^{-}, h_{2}^{-}\right\}\right\},\left\{\eta \in g_{1} \cap g_{2}: \eta \leqslant \min \left\{g_{1}^{+}, g_{2}^{+}\right\}\right\}\right), \\
& d_{1} \cap d_{2}=\left(\left\{\gamma \in h_{1} \cap h_{2}: \gamma \leqslant \min \left\{h_{1}^{+}, h_{2}^{+}\right\}\right\},\left\{\eta \in g_{1} \cup g_{2}: \eta \geqslant \max \left\{g_{1}^{-}, g_{2}^{-}\right\}\right\}\right) .
\end{aligned}
$$

We can use these operations to define:

Definition 9. Let $D_{1}=\left(d_{1}^{1}, \ldots, d_{m}^{1}\right), D_{2}=\left(d_{1}^{2}, \ldots, d_{m}^{2}\right)$ be two DEHFEs of degree $m$. Then, $D_{1} \cup D_{2}=$ $\left(d_{1}^{1} \cup d_{1}^{2}, \ldots, d_{m}^{1} \cup d_{m}^{2}\right)$ is their union, and $D_{1} \cap D_{2}=\left(d_{1}^{1} \cap d_{1}^{2}, \ldots, d_{m}^{1} \cap d_{m}^{2}\right)$ is their intersection. 
Example 3. With respect to the DEHFS $D_{2}^{\mathbf{X}}$ of degree 2 on $\mathbf{X}=\{x, y\}$ defined in Example 2,

$$
\begin{array}{r}
\left(D_{2}^{\mathbf{X}}\right)^{c}=\{\langle x,(\{0.3,0.5\},\{0.1,0.3,0.4\}),(\{0.1,0.2,0.4\},\{0.2,0.5\})\rangle, \\
\langle y,(\{0.4,0.5,0.6\},\{0.2,0.3\}),(\{0.1,0.2\},\{0.3,0.4,0.5\})\rangle\}
\end{array}
$$

\subsection{Comparison of Dual Extended Hesitant Fuzzy Elements}

We adhere to the comparison procedure in Section 2.3 to define a flexible procedure for the prioritization of typical DEHFEs with the same degree. The basic elements that we need are as follows:

Definition 10. Let $\omega=\left(\omega_{1}, \ldots, \omega_{m}\right)$ be a vector of numbers in $[0,1]$ such that $\omega_{1}+\ldots+\omega_{m}=1$. Let $D=\left(d_{1}, \ldots, d_{m}\right)$ be a DEHFE such that $d_{i}=\left(h_{i}, g_{i}\right)$ are typical DHFEs of degree $m$. With the help of Definition 6, we formulate

$$
s_{D}^{\omega}=\sum_{i=1, \ldots, m} w_{i} s_{d_{i}}, \quad p_{D}^{\omega}=\sum_{i=1, \ldots, m} w_{i} p_{d_{i}} .
$$

We say that $s_{D}^{\omega}$ is the $\omega$-score of the (typical) dual extended hesitant fuzzy element $D$, and $p_{D}^{\omega}$ is its w-accuracy function.

We are now ready to compare DEHFEs:

Definition 11. Let $\omega=\left(\omega_{1}, \ldots, \omega_{m}\right)$ be a vector of numbers in $[0,1]$ such that $\omega_{1}+\ldots+\omega_{m}=1$. Let $D_{1}=\left(d_{1}^{1}, \ldots, d_{m}^{1}\right), D_{2}=\left(d_{1}^{2}, \ldots, d_{m}^{2}\right)$ be typical DEHFEs of degree $m$.

(1) When either $s_{D_{1}}^{\omega}>s_{D_{2}}^{\omega}$ or $s_{D_{1}}^{\omega}=s_{D_{2}}^{\omega}$ and $p_{D_{1}}^{\omega}>p_{D_{2}}^{\omega}$ we say that $D_{1}$ is superior to $D_{2}$. We denote $D_{1} \succ D_{2}$.

(2) When $s_{D_{1}}^{\omega}=s_{D_{2}}^{\omega}$ and $p_{D_{1}}^{\omega}=p_{D_{2}}^{\omega}$, we say that $D_{1}$ is equivalent to $D_{2}$. We denote $D_{1} \sim D_{2}$.

Example 4. With $\omega=\left(\frac{1}{3}, \frac{2}{3}\right)$, let us compare the DEHFEs of degree 2:

$$
\begin{gathered}
D=(\{0.1,0.3,0.4\},\{0.3,0.5\}),(\{0.2,0.5\},\{0.1,0.2,0.4\})=\left(d_{1}, d_{2}\right) \\
D^{\prime}=(\{0.2,0.3\},\{0.4,0.5,0.6\}),(\{0.3,0.4,0.5\},\{0.1,0.2\})=\left(d_{1}^{\prime}, d_{2}^{\prime}\right)
\end{gathered}
$$

Some direct computations produce $s_{d_{1}}=\frac{0.1+0.3+0.4}{3}-\frac{0.3+0.5}{2} \approx 0.266, s_{d_{2}}=\frac{0.2+0.5}{2}-\frac{0.1+0.2+0.4}{3}=$ $\frac{0.7}{6} \approx 0.117, s_{d_{1}^{\prime}}=\frac{0.2+0.3}{2}-\frac{0.4+0.5+0.6}{3}=-0.25, s_{d_{2}^{\prime}}=\frac{0.3+0.4+0.5}{3}-\frac{0.1+0.2}{2}=0.25$.

Therefore, $s_{D}^{\omega}=\frac{1}{3} 0.266+\frac{2}{3} 0.116=0.1667$ and $s_{D^{\prime}}^{\omega}=\frac{1}{3}(-0.25)+\frac{2}{3} 0.25=0.083$.

We conclude that $D$ is superior to $D^{\prime}$ (i.e., $D \succ D^{\prime}$ ).

\section{Approach to Dual Extended Hesitant Fuzzy MADM Based on Weight Score Function}

In this section, we define the structure of the problems where the alternatives are described in terms of our newly defined notions. We also propose a decision-making mechanism that is adaptable, because it relies on the flexible concept of weights (associated with the relevant characteristics). Then, we illustrate the procedure with an example, and, finally, we check for its effectiveness.

\subsection{Problem Description}

Let $A=\left\{A_{1}, A_{2}, \cdots, A_{m}\right\}$ be a discrete set of alternatives, $C=\left\{C_{1}, C_{2}, \cdots, C_{n}\right\}$ be a collection of $n$ attributes, and $W=\left\{w_{1}, w_{2}, \cdots, w_{n}\right\}$ be a weight vector assigned to the attributes by the decision makers with the standard constraints $w_{j} \in[0,1], \sum_{j=1}^{n} w_{j}=1$. We assume that the global evaluation of the alternatives with respect to attributes is represented by a dual extended hesitant fuzzy matrix $\mathbf{R}=\left(r_{i j}\right)_{m \times n}$. By this we mean that the values associated with the alternatives for the modelization of MADM problems can be shown as in Table 1. 
Table 1. A general dual extended hesitant fuzzy MADM matrix R. The elements of the cells are DEHFEs.

\begin{tabular}{llclc}
\hline & $C_{1}$ & $C_{2}$ & $\cdots$ & $C_{n}$ \\
\hline$A_{1}$ & $r_{11}$ & $r_{12}$ & $\cdots$ & $r_{1 n}$ \\
$A_{2}$ & $r_{21}$ & $r_{22}$ & $\cdots$ & $r_{2 n}$ \\
$\vdots$ & $\vdots$ & $\vdots$ & $\ddots$ & $\vdots$ \\
$A_{m}$ & $r_{m 1}$ & $r_{m 2}$ & $\cdots$ & $r_{m n}$ \\
\hline
\end{tabular}

The next subsection formalizes the flexible decision methodology that benefits from weight score functions, a concept that we introduce to give a comprehensive evaluation of the alternatives.

\subsection{The Weight Score Function Method}

To make decisions in the setting described above, the following algorithm is self-explanatory:

Observe that Equation (8) defines a weight score function $R: A \longrightarrow[0,1]$. At the end of the process, any alternative such that $A_{i}=\max _{j=1,2, \cdots, m} R\left(A_{i}\right)$ can be recommended.

We proceed to illustrate the steps of this decision-making procedure by an example.

Example 5. Assume that there are four companies $A=\left\{A_{1}, A_{2}, A_{3}, A_{4}\right\}$ to be considered for the assessment of big data industry. The expert panel chooses the decision attribute set $C=\left\{C_{1}, C_{2}, C_{3}\right\}$ to be $C_{1}$ (denoted as Volume), $C_{2}$ (denoted as Variety) and $C_{3}$ (denoted as Velocity). Based on the characteristics of the big data industry, we can determine that all these attributes are desirable attributes. Suppose that the experts agree on the following set of weights according to their prior experience or expertise: $w=\left(w_{1}, w_{2}, w_{3}\right)=(0.3,0.2,0.5)$. The assessments arising from questionnaire investigation allow the experts to construct a dual extended hesitant fuzzy matrix with its tabular form given by Table 2 .

In what follows, we utilize Algorithm 1 to select the most suitable big data company under dual extended hesitant fuzzy information:

Step 1: The input is already available, namely, the dual extended hesitant fuzzy matrix $\mathbf{R}=$ $\left(r_{i j}\right)_{m \times n}$, which is shown in Table 2, and the weights vector $w$.

Step 2: The score value $s_{i j}^{\omega}$ of each alternative $A_{i}$ under attribute $C_{j}$ is shown in Table 3.

Step 3: We calculate the assessment score of alternative $A_{i}$ as follows:

$$
R\left(A_{1}\right)=0.4167, R\left(A_{2}\right)=0.3483, R\left(A_{3}\right)=0.28, \quad R\left(A_{4}\right)=0.2484 .
$$

Step 4: The ranking ordering is $A_{1} \succ A_{2} \succ A_{3} \succ A_{4}$, hence $A_{1}$ is the optimal big data company.

\section{Algorithm 1 The weight score function mechanism}

1: Identify the alternatives and attributes, and then input the dual extended hesitant fuzzy matrix $\mathbf{R}=\left(r_{i j}\right)_{m \times n}$ which is shown in the format of Table 1.

Input the weigths associated with the set of relevant attributes.

2: Compute the $w$-score value of each alternative $A_{i}$ under attribute $C_{j}$ by Definition 10 , for each possible $i$ and $j$. We denote it as $s_{i j}^{\omega}$.

3: Calculate the assessment score of alternative $A_{i}$ by the following expression, which produces its weight score:

$$
R\left(A_{i}\right)=\sum_{j=1}^{n} w_{j} \cdot s_{i j}^{\omega}
$$

4: Select the optimal alternative(s) by maximization of their weight scores, i.e., any alternative whose weight score $R\left(A_{i}\right)$ is at least as great as any other alternative's can be chosen. 
Table 2. The dual extended hesitant fuzzy MADM matrix in Example 5.

\begin{tabular}{ccccc}
\hline & $C_{1}$ & $C_{2}$ & $C_{3}$ \\
\hline$A_{1}$ & $<(\{0.7,0.8\},\{0.1,0.2\}),(\{0.6,0.7\},\{0.1,0.3\})>$ & $<(\{0.6,0.8\},\{0.1,0.2\}),(\{0.6,0.7\},\{0.1,0.2\})>$ & $<(\{0.6,0.7\},\{0.1,0.3\}),(\{0.5,0.7\},\{0.1,0.3\})>$ \\
$A_{2}<(\{0.6,0.7\},\{0.1,0.3\}),(\{0.5,0.7\},\{0.2,0.3\})>$ & $<(\{0.6,0.7\},\{0.1,0.3\}),(\{0.5,0.7\},\{0.1,0.3\})>$ & $<(\{0.5,0.6\},\{0.1,0.4\}),(\{0.5,0.6\},\{0.2,0.3\})>$ \\
$A_{3}<(\{0.5,0.6\},\{0.2,0.3\}),(\{0.5,0.6\},\{0.2,0.3\})>$ & $<(\{0.5,0.7\},\{0.1,0.3\}),(\{0.4,0.7\},\{0.1,0.3\})>$ & $<(\{0.3,0.6\},\{0.1,0.4\}),(\{0.5,0.6\},\{0.2,0.4\})>$ \\
$A_{4}<(\{0.4,0.5\},\{0.3,0.4\}),(\{0.3,0.4\},\{0.3,0.4\})>$ & $<(\{0.3,0.5\},\{0.1,0.5\}),(\{0.3,0.7\},\{0.1,0.3\})>$ & $<(\{0.3,0.5\},\{0.1,0.4\}),(\{0.5,0.6\},\{0.3,0.4\})>$ \\
\hline
\end{tabular}

Table 3. The score function matrix in Example 5.

\begin{tabular}{cccc}
\hline & $C_{1}$ & $C_{2}$ & $C_{3}$ \\
\hline$A_{1}$ & 0.5000 & 0.5167 & 0.4167 \\
$A_{2}$ & 0.3833 & 0.4167 & 0.3000 \\
$A_{3}$ & 0.3000 & 0.3667 & 0.2333 \\
$A_{4}$ & 0.3667 & 0.2333 & 0.1833 \\
\hline
\end{tabular}




\subsection{Effectiveness Test}

This section justifies the adequacy of Algorithm 1 for making decisions in the context of dual extended hesitant fuzzy information. Certain test criteria that were proposed by Wang and Triantaphyllou [17] are applied in the following terms:

Test Criterion 1: If we replace the decision values of a non-optimal alternative by those of the worst case, then the optimal alternative should not change.

Test Criterion 2: the MADM method should comply with the transitive property.

Test Criterion 3: When an appointed issue is disassembled into smaller ones and the same MADM method is employed, then the assorted ordering of the alternatives should be the same as the ranking of the original one.

In the next subsections, we confirm the verification of these test criteria for our developed dual extended hesitant fuzzy MADM method based on weight score function.

\subsubsection{Effectiveness Test by Criterion 1}

One can easily observe that Algorithm 1 verifies this criterion. The reason is that the assessment score of any alternative is independent of the structure of the other alternatives. Therefore, any exchange among the values of the remaining options still produces the highest evaluation at the same alternative.

For example, in the situation of Section 4.2, if we exchange the membership and non-membership degrees of alternatives $A_{2}, A_{3}$ (non-optimal option) and $A_{4}$ (worst option) in the matrix $P$, then the switched decision matrix turns into $P^{\prime}$, which is shown in Table 4.

According to the information above, the presented weight score function has been applied, and the optimal alternative is $A_{1}$ which is same as that of the original ranking. Therefore, the proposed algorithm verifies the test Criterion 1 .

\subsubsection{Effectiveness test by Criteria 2 and 3}

According to these tests, if we decompose the appointed problem into the sub-issues $\left\{A_{1}, A_{2}\right\}$, $\left\{A_{2}, A_{3}\right\},\left\{A_{3}, A_{4}\right\}$ and $\left\{A_{1}, A_{4}\right\}$ and the procedure steps of the algorithm are employed, then we obtain that the ranking of these smaller issues is $A_{1} \succ A_{2}, A_{2} \succ A_{3}, A_{3} \succ A_{4}$ and $A_{1} \succ A_{4}$, respectively. Hence, by combining Criteria 2 and 3 above, we obtain that the overall ranking order of the alternatives is $A_{1} \succ A_{2} \succ A_{3} \succ A_{4}$, which is equal to that of the original ranking order. Therefore, the developed algorithm agrees with the test Criteria 2 and 3. 
Table 4. The switched dual extended hesitant fuzzy matrix $P^{\prime}$ in Example 5.

$C_{1}$

$C_{2}$

$C_{3}$

$A_{1}<(\{0.7,0.8\},\{0.1,0.2\}),(\{0.6,0.7\},\{0.1,0.3\})><(\{0.6,0.8\},\{0.1,0.2\}),(\{0.6,0.7\},\{0.1,0.2\})><(\{0.6,0.7\},\{0.1,0.3\}),(\{0.5,0.7\},\{0.1,0.3\})>$

$A_{2}<(\{0.1,0.3\},\{0.6,0.7\}),(\{0.2,0.3\},\{0.5,0.7\})>\quad<(\{0.1,0.3\},\{0.6,0.7\}),(\{0.1,0.3\},\{0.5,0.7\})><(\{0.1,0.4\},\{0.5,0.6\}),(\{0.2,0.3\},\{0.5,0.6\})>$

$A_{3}<(\{0.2,0.3\},\{0.5,0.6\}),(\{0.2,0.3\},\{0.5,0.6\})>\quad<(\{0.1,0.3\},\{0.5,0.7\}),(\{0.1,0.3\},\{0.4,0.7\})>\quad<(\{0.1,0.4\},\{0.3,0.6\}),(\{0.2,0.4\},\{0.5,0.6\})>$

$A_{4}<(\{0.3,0.4\},\{0.4,0.5\}),(\{0.3,0.4\},\{0.3,0.4\})>\quad<(\{0.1,0.5\},\{0.3,0.5\}),(\{0.1,0.3\},\{0.3,0.7\})>\quad<(\{0.1,0.4\},\{0.3,0.5\}),(\{0.3,0.4\},\{0.5,0.6\})>$ 


\section{Conclusions}

The natural hybridization of two recent extensions of hesitant fuzzy sets produces a flexible framework of uncertain information whose operations are pretty natural. This model is called DEHFS (for dual extended hesitant fuzzy sets) and it subsumes successful concepts such as intuitionistic fuzzy sets, hesitant fuzzy sets, and extended hesitant fuzzy sets. We have summarized the relevant relationships in Figure 1. In conclusion, DEHFSs constitute a neat formulation that embodies uncertain or vague knowledge in a large variety of forms.

Further research will be needed to extend the algebra of the novel elements in our model. Distances, correlation coefficients and aggregation operators must be defined to meet the requirements of the practitioners. Extension principles [22,40] can provide basic operators and aggregation operations of DEHFSs as in Section 4 of [10]. Finally, these tools under development should enable the researchers to produce additional decision making mechanisms for this novel framework.

Author Contributions: The authors contributed equally to this work.

Funding: The research of G. Santos-García was funded by the Spanish MINECO projects TRACES TIN2015-67522-C3-3-R and Strongsoft TIN2012-39391-C04-04.

Conflicts of Interest: José Carlos R. Alcantud, Gustavo Santos-García, Xindong Peng, and Jianming Zhan declare that they have no conflict of interest.

\section{Abbreviations}

The following abbreviations are used in this manuscript:

$\begin{array}{ll}\text { MADM } & \text { Multi-attribute decision making } \\ \text { HFS } & \text { Hesitant fuzzy set } \\ \text { DHFS } & \text { Dual hesitant fuzzy set } \\ \text { HFE } & \text { Hesitant fuzzy element } \\ \text { T-HFE } & \text { Typical HFE } \\ \text { EHFS } & \text { Extended hesitant fuzzy set } \\ \text { XHFS } & \text { Expanded hesitant fuzzy set } \\ \text { EHFE } & \text { Extended hesitant fuzzy element } \\ \text { DHFS } & \text { Dual hesitant fuzzy set } \\ \text { T-DHFS } & \text { Typical dual hesitant fuzzy set } \\ \text { DHFE } & \text { Dual hesitant fuzzy element } \\ \text { T-DHFE } & \text { Typical DHFE } \\ \text { DEHFS } & \text { Dual extended hesitant fuzzy set } \\ \text { DEHFE } & \text { Dual extended hesitant fuzzy element } \\ \text { IFS } & \text { Intuitionistic fuzzy set }\end{array}$

\section{References}

1. Zadeh, L. Fuzzy sets. Inf. Control 1965, 8, 338-353. [CrossRef]

2. Zhan, J.; Sun, B.; Alcantud, J.C.R. Covering based multigranulation (I, T)-fuzzy rough set models and applications in multi-attribute group decision-making. Inf. Sci. 2019, 476, 290-318. [CrossRef]

3. Zhan, J.; Ali, M.I.; Mehmood, N. On a novel uncertain soft set model: Z-soft fuzzy rough set model and corresponding decision making methods. Appl. Soft Comput. 2017, 56, 446-457. [CrossRef]

4. Ma, X.; Liu, Q.; Zhan, J. A survey of decision making methods based on certain hybrid soft set models. Artif. Intell. Rev. 2017, 47, 507-530. [CrossRef]

5. Zhang, H.; Zhan, J.; He, Y. Multi-granulation hesitant fuzzy rough sets and corresponding applications. Soft Comput. 2019. [CrossRef]

6. Bustince, H.; Barrenechea, E.; Pagola, M.; Fernandez, J.; Xu, Z.; Bedregal, B.; Montero, J.; Hagras, H.; Herrera, F.; De Baets, B. A historical account of types of fuzzy sets and their relationships. IEEE Trans. Fuzzy Syst. 2016, 24, 179-194. [CrossRef]

7. Torra, V. Hesitant fuzzy sets. Int. J. Intell. Syst. 2010, 25, 529-539. [CrossRef] 
8. Alcantud, J.C.R.; Giarlotta, A. Necessary and possible hesitant fuzzy sets: A novel model for group decision making. Inf. Fusion 2019, 46, 63-76. [CrossRef]

9. Atanassov, K.T. Intuitionistic fuzzy sets. Fuzzy Sets Syst. 1986, 20, 87-96. [CrossRef]

10. Zhu, B.; Xu, Z.; Xia, M. Dual hesitant fuzzy sets. J. Appl. Math. 2012, 2012, 879629. [CrossRef]

11. Alcantud, J.C.R.; de Andrés Calle, R.; Torrecillas, M. Hesitant fuzzy worth: An innovative ranking methodology for hesitant fuzzy subsets. Appl. Soft Comput. 2016, 38, 232-243. [CrossRef]

12. Herrera, F.; Martínez, L.; Torra, V.; Xu, Z. Hesitant fuzzy sets: An emerging tool in decision making. Int. J. Intell. Syst. 2014, 29, 493-944. [CrossRef]

13. Rodríguez, R.; Martínez, L.; Torra, V.; Xu, Z.; Herrera, F. Hesitant fuzzy sets: State of the art and future directions. Int. J. Intell. Syst. 2014, 29, 495-524. [CrossRef]

14. Xu, Z. Hesitant Fuzzy Sets Theory; Studies in Fuzziness and Soft Computing; Springer: Berlin/Heidelberg, Germany, 2014; Volume 314.

15. Alcantud, J.C.R.; de Andrés, R. A segment-based approach to the analysis of project evaluation problems by hesitant fuzzy sets. Int. J. Comput. Int. Syst. 2016, 29, 325-339. [CrossRef]

16. Zhu, B.; Xu, Z. Extended hesitant fuzzy sets. Technol. Econ. Dev. Econ. 2016, 22, 100-121. [CrossRef]

17. Wang, X.; Triantaphyllou, E. Ranking irregularities when evaluating alternatives by using some ELECTRE methods. Omega 2008, 36, 45-63. [CrossRef]

18. Xia, M.; Xu, Z. Hesitant fuzzy information aggregation in decision making. Int. J. Approx. Reason. 2011, 52, 395-407. [CrossRef]

19. Farhadinia, B. A series of score functions for hesitant fuzzy sets. Inf. Sci. 2014, 277, 102-110. [CrossRef]

20. Farhadinia, B. A multiple criteria decision making model with entropy weight in an interval-transformed hesitant fuzzy environment. Cogn. Comput. 2017, 9, 513-525. [CrossRef]

21. Bedregal, B.; Reiser, R.; Bustince, H.; López-Molina, C.; Torra, V. Aggregation functions for typical hesitant fuzzy elements and the action of automorphisms. Inf. Sci. 2014, 255, 82-99. [CrossRef]

22. Alcantud, J.C.R.; Torra, V. Decomposition theorems and extension principles for hesitant fuzzy sets. Inf. Fusion 2018, 41, 48-56. [CrossRef]

23. Rodríguez, R.; Bedregal, B.; Bustince, H.; Dong, Y.; Farhadinia, B.; Kahraman, C.; Martínez, L.; Torra, V.; $\mathrm{Xu}, \mathrm{Y}$;; $\mathrm{Xu}, \mathrm{Z}$; ; et al. A position and perspective analysis of hesitant fuzzy sets on information fusion in decision making. Towards high quality progress. Inf. Fusion 2016, 29, 89-97. [CrossRef]

24. Rodríguez, R.M.; Martínez, L.; Herrera, F.; Torra, V. A review of hesitant fuzzy sets: Quantitative and qualitative extensions. In Fuzzy Logic in Its 50th Year: New Developments, Directions and Challenges; Kahraman, C., Kaymak, U., Yazici, A., Eds.; Springer: Berlin/Heidelberg, Germany, 2016; pp. 109-128.

25. Wang, F.; Li, X.; Chen, X. Hesitant fuzzy soft set and its applications in multicriteria decision making. J. Appl. Math. 2014, 2014, 643785. [CrossRef]

26. Li, X.; Chen, X. D-Intuitionistic hesitant fuzzy sets and their application in multiple attribute decision making. Cogn. Comput. 2018, 10, 496-505. [CrossRef]

27. Xu, Z.; Zhou, W. Consensus building with a group of decision makers under the hesitant probabilistic fuzzy environment. Fuzzy Optim. Decis. Mak. 2017, 16, 481-503. [CrossRef]

28. Li, J.; Wang, J.Q. Multi-criteria outranking methods with hesitant probabilistic fuzzy sets. Cogn. Comput. 2017, 9, 611-625. [CrossRef]

29. Alcantud, J.C.R.; Santos-García, G. Expanded hesitant fuzzy sets and group decision making. In Proceedings of the 2017 IEEE International Conference on Fuzzy Systems (FUZZ-IEEE), Naples, Italy, 9-12 July 2017; pp. 1-6.

30. Zhu, B.; Xu, Z. Some results for dual hesitant fuzzy sets. J. Intell. Fuzzy Syst. 2014, 26, 1657-1668.

31. $\mathrm{Xu}, \mathrm{Z}$; Y Yager, R.R. Some geometric aggregation operators based on intuitionistic fuzzy sets. Int. J. Gen. Syst. 2006, 35, 417-433. [CrossRef]

32. Lu, N.; Liang, L. Correlation coefficients of extended hesitant fuzzy sets and their applications to decision making. Symmetry 2017, 9, 47. [CrossRef]

33. Ye, J. Correlation coefficient of dual hesitant fuzzy sets and its application to multiple attribute decision making. Appl. Math. Model. 2014, 38, 659-666. [CrossRef]

34. Wang, L.; Ni, M.; Zhu, L. Correlation measures of dual hesitant fuzzy sets. J. Appl. Math. 2013, $2013,593739$. [CrossRef] 
35. Su, Z.; Xu, Z.; Liu, H.; Liu, S. Distance and similarity measures for dual hesitant fuzzy sets and their applications in pattern recognition. J. Intell. Fuzzy Syst. 2015, 29, 731-745. [CrossRef]

36. Chen, J.; Huang, X.; Tang, J. Distance measures for higher order dual hesitant fuzzy sets. J. Comp. Appl. Math. 2018, 37, 1784-1806. [CrossRef]

37. Wang, H.; Zhao, X.; Wei, G. Dual hesitant fuzzy aggregation operators in multiple attribute decision making. J. Intell. Fuzzy Syst. 2014, 26, 2281-2290.

38. Peng, X.; Yang, Y. Research on dual hesistant fuzzy soft set. Comput. Eng. 2015, 41, 262-267.

39. Garg, H.; Arora, R. Dual hesitant fuzzy soft aggregation operators and their application in decision-making. Cogn. Comput. 2018, 10, 769-789. [CrossRef]

40. Torra, V.; Narukawa, Y. On hesitant fuzzy sets and decision. In Proceedings of the 2009 IEEE International Conference on Fuzzy Systems (FUZZ-IEEE), Jeju Island, Korea, 20-24 August 2009; pp. 1378-1382.

(C) 2019 by the authors. Licensee MDPI, Basel, Switzerland. This article is an open access article distributed under the terms and conditions of the Creative Commons Attribution (CC BY) license (http:// creativecommons.org/licenses/by/4.0/). 